\title{
Path, process and (a)telicity in space: Motion predicates in LSCu, Sign Language of Cuba
}

\author{
Alicia Calderón Verde (Universidad Tecnológica de La Habana), \\ Donny Wilson Limonta, Gilma Cervantes Soliño, \\ Ariel Hernández Hernández (CENDSOR, Cuba), \\ Elena E. Benedicto (IELLab, Purdue University) \\ acalderon@arquitectura.cujae.edu.cu, ansoccendsor@infomed.sld.cu \\ (Wilson \& Hernández), ebenedi@purdue.edu
}

\begin{abstract}
This paper examines the morphological properties of NMM for [mouth] and [eyegaze] and their association with the syntactic realization of the predicate's subeventive structure. Two different NMM-[mouth] are identified, one for the process subevent ([uu]) and another for the telic subevent ([ph]). The systematic presence of these NMM-[mouth] as grammatical markers of subeventive structure confirms initial findings by Benedicto, Branchini, and Mantovan (2015) in favor of a deconstructivist approach to the predicate subeventive structure. NMM-[eyegaze] is shown to be associated with the argument of the respective subeventive structure: the Undergoer for the process subevent and the EndPoint for the telic subevent.
\end{abstract}

Keywords: non-manual markers, syntax-semantics interface, telicity, subeventive structure, serial verb construction

\section{Introduction}

This study is part of a larger, cross-linguistic and cross-modality project on motion predicates, exploring the syntactic representation of a predicate's subeventive structure. It originally involved 9 spoken languages (from different language families in Central America, Africa and Asia) and 3 sign languages (American Sign Language: ASL, Italian Sign Language: LIS, and Hong Kong Sign Language: HKSL) to which LSCu (Sign Language of Cuba) has since been added.

The specific goal of the present paper is to examine the morphosyntactic status of two non-manual markers (NMM), eye gaze and mouth, in motion predicates in LSCu. We want to evaluate their contribution to sub-eventive structure, in particular to telicity, within the complex structure of a motion predicate.

The larger issue this paper contributes to concerns the conceptualization of what has been called Inner Aspect or Aktionsart and, in general, the representation of a predicate's sub-eventive structure. Vendler (1957) established 4 categories of verbs according to their Aktionsart: states, activities, accomplishments and achievements. Leaving states aside, activities were supposed to represent an event with just a process, while accomplishments, 
which by definition have a telos, add an end point to that process. In that sense, activities have only one subeventive component (the process) while accomplishments have two subeventive components (a process and a telos or end point), and so the term telic is also applied to them. ${ }^{1}$

The general discussion has centered around whether these distinctions are encoded lexically in the verbal units or in the syntax (in the structural representation) of the predicates. ${ }^{2}$ The lexicalist position proposed that verbal units are marked in the lexicon as either activities or accomplishments. However, starting in the 90's, research began to focus on the syntactic devices that could manipulate whether a predicate could be considered an activity or an accomplishment. ${ }^{3}$ Out of that body of research came theoretical analyses that proposed that verbal units are not specified one way or the other in the lexicon and that, as predicates are being built up in the syntax in the usual combinatorial way, the specific interpretation of a predicate (as either an activity with only a process, or as an accomplishment with both a process and a telos) can be calculated out of their particular functional configurational structure. These latter positions, with several degrees of variation among them, are represented by, e.g., the works of Borer (2005) and Ramchand (2008), among many others. ${ }^{4}$

As part of the larger project on motion predicates mentioned above, we have been able to observe the subeventive decomposition of motion predicates in the shape of Serial Verb Constructions (SVCs). SVCs encoding subeventive components (process and telos) have been seen in Spoken Languages (e.g., Mandarin, Ghanaian Student Pidgin, GSP):

(1) yi zhi niao fei guo qu dao liba shang.

one CL bird fly cross go arrive fence top

'A bird flew over (all the way) to the top of the fence.' [Mandarin; Chen 2018]

\footnotetext{
${ }^{1}$ An example of an activity predicate is (i-a). which denotes the ongoing process of eating a contextually relevant apple, whereas (i-b) contains a process (the eating of the apple) as well as a telos (the end point of the apple-eating activity where there is no more apple), therefore qualifying as an accomplishment or telic predicate. The examples in (ii) reflect the same contrast within a motion predicate.
}
(i) a. John was eating the apple.
(ii) a. John was crossing the street.
b. John ate the apple.
b. John crossed the street.

${ }^{2}$ Dowty (1979), who generalized the use of a battery of tests like for an hour/in an hour for activities vs accomplishments, recognized that these inner aspect properties are properties of predicates, but kept using verbs throughout his work ("In this section I will introduce a classification of verbs (or rather, of verb phrases) that...” Dowty 1979, 51, our emphasis).

${ }^{3}$ The usual culprits are the definiteness of the object and the (im)perfectivity of the verbal unit. The examples in (i) in footnote 1, illustrate the latter: the imperfective -ing makes (i-a) an activity (as opposed to (i-b), which is an accomplishment). The examples in (iii) illustrate how an indefinite (iii-b) can trigger an activity reading (detected by for an hour).

(iii) a. John ate the apple (*for an hour).

b. John ate an apple (for an hour).

${ }^{4}$ These latter positions that 'generate' the subeventive meaning out of the structure are referred to as constructionist (Borer), generative-constructivist (Ramchand) or similar terms; though different in the specifics of their technical approach, they share the view that the structure is generated out of a combinatorial system (the syntax). These are not to be confused with Construction Grammar, which though sharing the relevance of the structure (the construction) does not conceive it in a 'generative' way, as a result of a combinatorial system (but as a closed element to be listed in the lexicon).

Chapter 1 of Ramchand (2008) provides a good concise context for these two views (the lexicalist/ projectionist and generative-constructivist), whereas Chapter 1 of Borer (2005) provides an in-depth view on a wider set of issues. 
(2) The bird fly pass the river top go catch the tree top

$\mathrm{D}$ bird fly pass $\mathrm{D}$ river top go $\mathrm{REACH} \mathrm{D}$ tree top

'The bird flew across the river (all the way) to the tree top.' [GSP; Osei-Tutu 2016]

In both cases in (1-2), we can see an overt head encoding telicity (the semi-grammaticalized heads dao in (1) and catch in (2)), following the process subcomponent (represented by the path units guo qu 'cross go' and pass...go 'go across', respectively). The second component, encoding telicity, is absent in atelic utterances (where only the process path is present, as expected).

SVCs in Sign Languages have been reported for a variety of purposes (see Supalla 1990; Lau 2012; Benedicto, Cvejanov, and Quer 2008). For motion predicates, and more specifically encoding subeventive structure, they have been seen in ASL, HKSL and LIS. Here is an example in LIS (from Benedicto, Branchini, and Mantovan 2015): ${ }^{5}$

(3) $\quad$ FLY ... $\frac{\text { FF }}{1+\text { GO.X:y }} \frac{\text { PHP }}{\text { V]+REACH.y }}$

FLY ... 5)+BE-AT.y-

'... (the bird) flies (all the way [from location $x$ ]) up to (the tree at) [location $y$ ]'

In (3), as in (1-2) above, we can see a final telic subcomponent, in the form of the classifier form $\mathrm{V}]+\mathrm{REACH}$, following a process path in the form of another classifier form 1+GO. Each co-occurs with dedicated NMM ([PHP] and [FF], respectively). The existence of these structures analytically deconstructing the subeventive components of the predicate, both in spoken and in sign languages, provides support for the view that telicity (and thus, by extension, Aktionsart) is built up in the syntax.

The main goal of this paper is to provide further evidence for the hypothesis that the subeventive structure of predicates is represented and built up in the syntax component of language, following constructionist or generative-constructivist approaches developed within the framework of Generative Grammar (e.g., Borer 2005; Ramchand 2008; see footnote 4).

The structural Hypothesis that we work with in this paper is the one in (4) below. It was developed out of structures such as those exemplified in (3) above and it is based on an analysis of SVC along the lines of Larson (1991) and subsequent works (Carstens 2002; Collins 2002; Aboh 2009) ${ }^{6}$ and, specifically for sign languages, on the work in Benedicto, Branchini, and Mantovan (2015) for LIS. In particular, the structures in (4) propose two recursively embedded sub-structures under $\nu^{\circ}: \pi$ and $\tau$, whose heads are circled. Substructure $\pi$, encoding 3D-Complex Path (which includes specifications for the 3 dimensions of space: horizontal, vertical, sagittal/deictic) together with $v^{\circ}$ provides the process interpretation of the predicate; this corresponds, in (3), to 1+GO.x:y with NMM-[FF]. Substructure $\tau$, encoding telicity, includes an XP.loc constituent and a $\tau$ head, whose manual content is dubbed REACH (corresponding to V]+REACH.y with NMM-[PHP] in (3)); this head interprets the XP.loc as an

\footnotetext{
${ }^{5}$ The notation used in this paper includes one line for each hand (H1 and H2). Classifier predicates are represented with a letter or number indicating the handshape $(1, \mathrm{~V}])$, followed by a ' + ' sign and a gloss describing the specific phonetic/kinetic movement associated with the classifier predicate. The diacritics ] and ) mean, respectively, bending at the second phalanges and curved shape. Subindices $(x, y)$ are used to indicate locations in space. ' $x: y$ ' means from location $x$ to location $y$; ' $x$ :/y' means from location $x$ towards location $y$.

${ }^{6}$ The main contribution of Larson's work, and a substantial one at that, was to show that SVCs had an underlying recursive complement structure, not a coordination or an adjunction structure, alternatives that were being considered at the time. Collins' and Aboh's work further show that SVCs may not be simple recursion of VPs, but rather that further functional structure may be involved; Carstens' addresses issues of linearization of SVCs in head-final vs head-initial languages.
} 
EndPoint (that is, the location denoted by XP.loc has been reached), thus yielding a telic interpretation.

Under this view of subeventive predicate structure, telic predicates have an underlying structure with two subeventive heads ( $\pi$ and $\tau$ ) corresponding to the process subevent and the telic (EndPoint) subevent (as in $4 \mathrm{a}$ ), while non-telic predicates lack the $\tau$-substructure and have, thus, only the process subevent component, as in (4b). In this latter case, if there is an XP.loc (which would be optional), it ends up being interpreted as a potential 'towards' Goal, but not as EndPoint (which, under this Hypothesis, is only accomplished via the $\tau$ head).

(4) Structural decomposition of motion predicates sub-eventive structure (Benedicto, Branchini, and Mantovan 2015)

a.

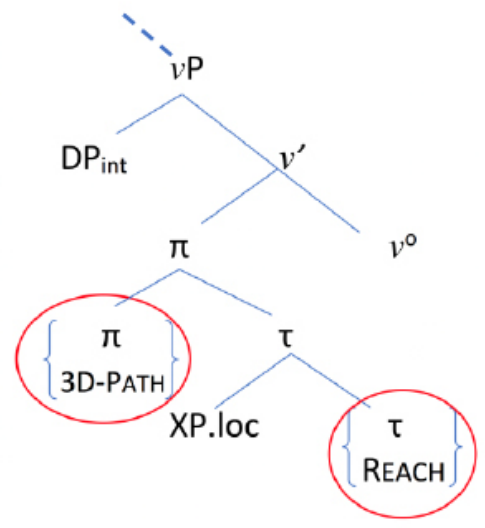

b.

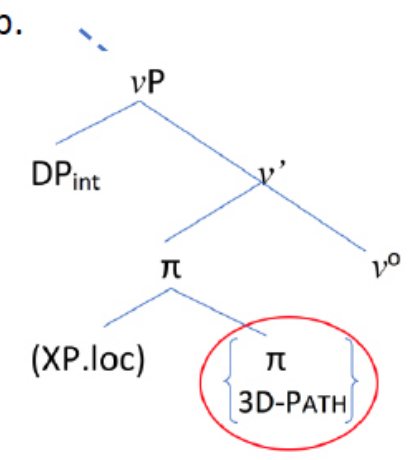

In this paper we will be showing that there are certain NMMs in LSCu that systematically correlate with the structure above, in particular:

(5) 1. The process sub-event encoding the 3D-Complex Path correlates with a [u] NMM-[mouth] gesture with optional release of air;

2. The telic sub-event correlates with a [ph] NMM-[mouth] mouth gesture co-timed with the Endpoint of the motion vector;

3. A NMM-[eyegaze] is associated with the DPint argument (the Figure or Undergoer) at the beginning of the Path articulation;

4. An additional NMM-[eyegaze] appears associated with the Endpoint location of the motion vector.

Our hypothesis for this paper is that those NMMs constitute the morphological realization of the subeventive structure in (4), in particular:

(6) a. the NMM-[mouth] [u] and [ph] in (5-1) and (5-2) are part of the morphological matrix of heads $\pi$ and $\tau$, respectively and

b. the [eyegaze]-NMMs are, following Neidle et al. (2000), agreement markers. ${ }^{7}$

The structural representation, thus, ends up as in (7), with square numbers referring to (5):

\footnotetext{
${ }^{7}$ Though the specifics of their technical implementation has been challenged (e.g., by Thompson, Emmorey, and Kluender 2006), the original idea conceptualizing [eyegaze] as agreement goes back, to the best of our knowledge, to Neidle et al. (2000).
} 
(7)

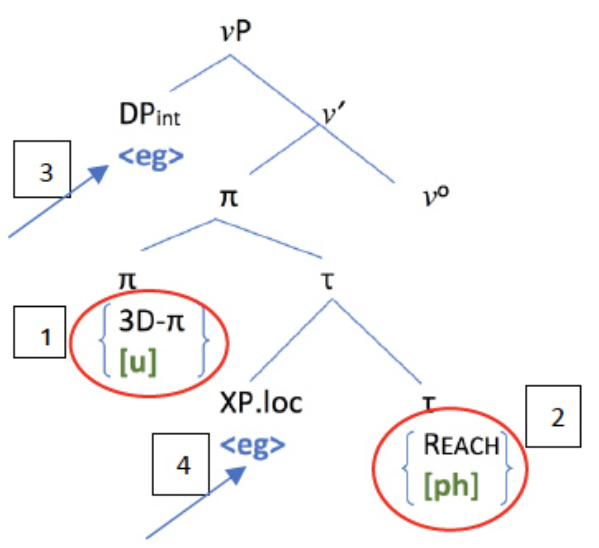

\section{Background on LSCu}

La Lengua de Señas Cubana, LSC(u), ${ }^{8}$ is the language used by the Deaf community in Cuba. Though the origins of the language are not well-known, it is accepted that around 1924 the first manual alphabet, AMA, was introduced by a Spanish teacher. The first Deaf School also dates from that time. Up until 1992, an oralist tradition was followed in the school system. That changed in 1994 with the introduction of LSC(u) in Deaf Schools, under agreement with the ANSOC (Asociación Nacional de Sordos de Cuba, the National Deaf Association of Cuba) and the Ministry of Education. From 2006 to 2010, the first Bachelor's Degree in LSC(u) was created with an approach combining Linguistics and Interpretation.

The CENDSOR (Centro para el Desarrollo del Sordo, Center for the Development of the Deaf) is a Research Center, within the structure of the ANSOC, that became active in 2008. Since then, it has been involved in linguistic research addressing phonological and morphological studies as well as general syntactic description of the language; examples include studies on the characterization of the phonological parameters of LSC(u), on the phonological and morphological properties of movement (Calderón 2013), on aspect, on the determiner system, among other topics. It also provides support for the teaching of LSC(u) and the training of Deaf instructors and interpreters, as well as the production of audiovisual material. It coordinates teams of Deaf researchers and instructors throughout the Cuban territory (see (8)).

\footnotetext{
${ }^{8}$ The acronym used in Cuba is LSC. Since the same acronym is used internationally for other sign languages (e.g., Sign Language of Colombia, Catalan Sign Language), we have chosen to add a -u at the end of the acronym: LSCu. In this section, we have chosen to maintain the $-\mathrm{u}$ in parenthesis to respect the original use when providing the local background to the language.
} 
(8) CENDSOR teams in Cuba

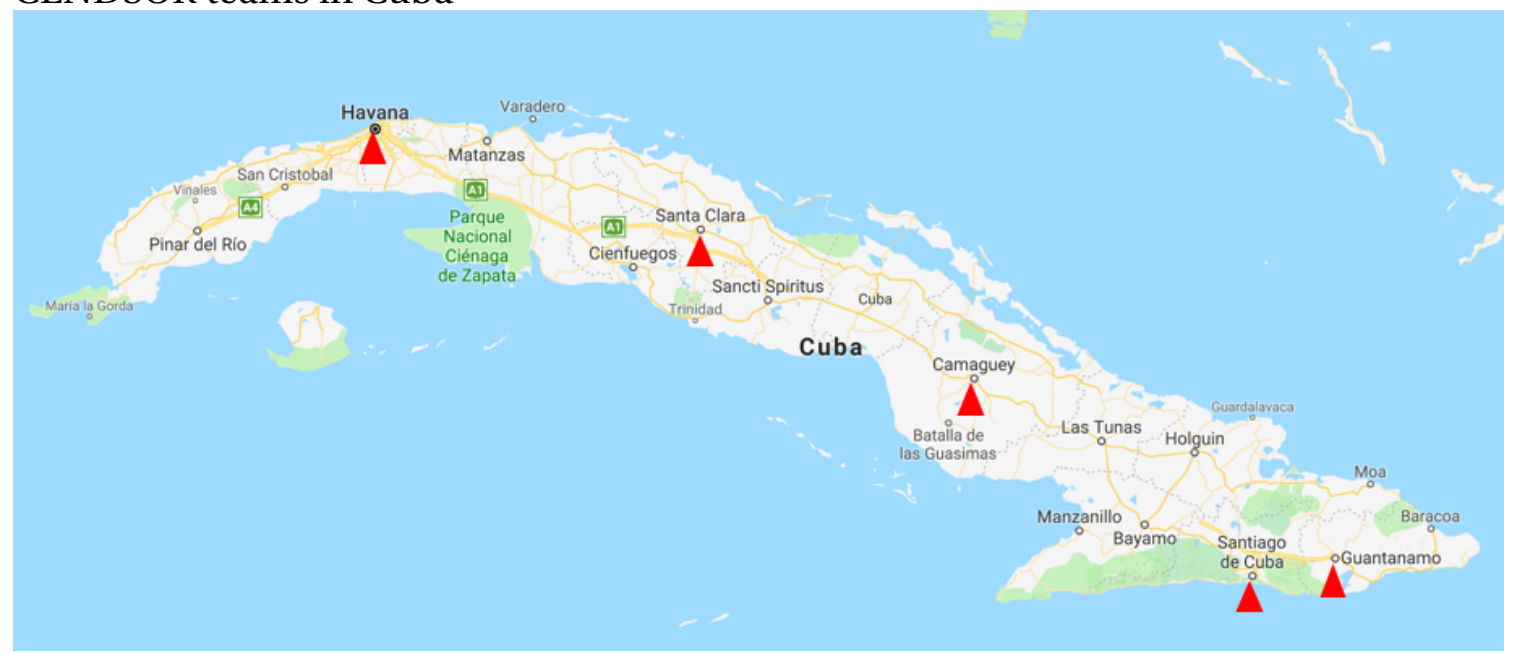

\section{Methodology}

\subsection{Data collection}

The data for this work were collected using a self-paced application (Benedicto 2017) designed at Purdue University in collaboration with the Envision Center. The application contains 175 video animations randomly distributed into 7 blocks. The video animations encode variables for several parameters related to motion predicates (including 3D Complex Path, process, telicity, result and agentivity). Data were obtained from 3 adult native signers, fluent users of LSCu. Each signer was asked to produce 2 renderings of each video animation, consecutively. ${ }^{9}$ A total of 1050 productions was obtained. A qualitative follow-up with the signers was conducted, as needed for clarification or deeper understanding. Two cameras were used for the recording, one with a frontal view and another at a side angle. The two recordings were synchronized and clipped:

(9)

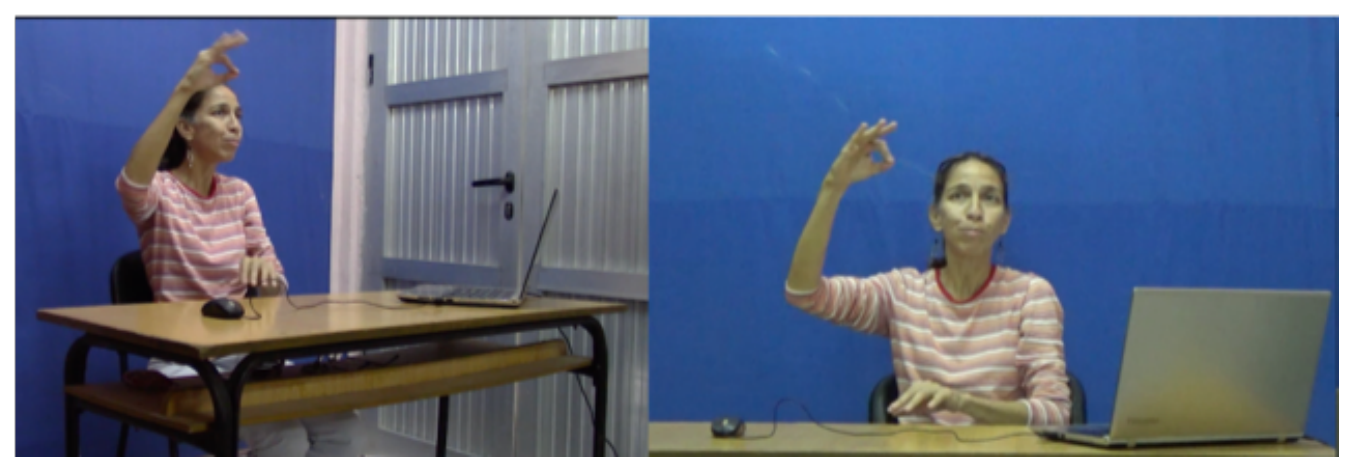

The synchronized clips were processed in ELAN with coding tiers for: H1 and H2 (dominant and non-dominant hand); NMM-eyegaze; NMM-mouth. Spatial coding for Figure, Endpoint and Movement Vectorization (3D-Complex Path) was used according to the categorical specifications in Benedicto, Branchini, and Mantovan (2015).

\footnotetext{
${ }^{9}$ They were also asked to sign the ID number of video animation at the beginning of each rendering.
} 


\subsection{Data transcription and glossing}

The above information is organized in the glossing of our examples for this paper in two lines corresponding to $\mathrm{H} 1$ (dominant) and $\mathrm{H} 2$ (non-dominant hand), a third line for English morphological glosses and a translation line, ${ }^{10}$ with additional coding as described for (10) below:

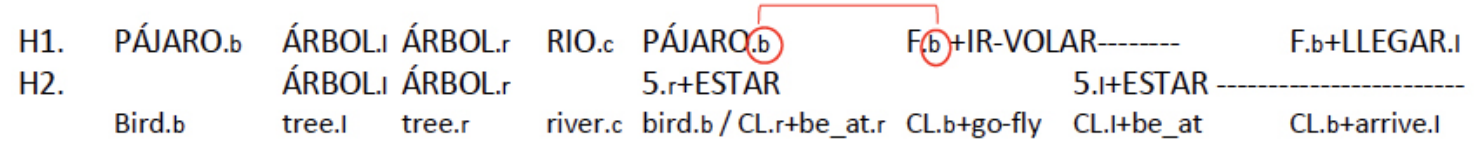

'There is a bird, a tree on the left, a tree on the right, a river in the middle; the bird, at the tree on the right, flies across the river all the way up to the tree on the left.'

Each argument (e.g., PÁJARO, ÁRBOL, etc. ...) is followed by a referential index (e.g., .b for PÁJARO , .c for RIO, ...) used for co-referential purposes (e.g., between an argument and a classifier handshape, as indicated by the red line in (10)). Classifier predicates are transcribed in two parts separated by a '+' sign; the first part encodes the handshape using the alphabet letter closest to its shape ${ }^{11}$ (e.g., $\mathrm{F}+$ ) and the second part uses a transcription of the lexical meaning of the predicate (roughly corresponding to the kinetic properties of the sign). The transcription of $\mathrm{H} 1$ and $\mathrm{H} 2$ is time-aligned (e.g., PÁJARO and 5.r+ESTAR are co-articulated). If a handshape is held in time, it is signaled with a continuous line (e.g., —

\section{NMM-[mouth] patterns}

In the data collected, two NMM-[mouth] gestures were identified, showing morphological value: one with joined protruding lips transcribed as [uu], with optional continuous release of air, and one involving buildup of air in the mouth with sudden release, transcribed as [ph]. They appear illustrated in (11) below:
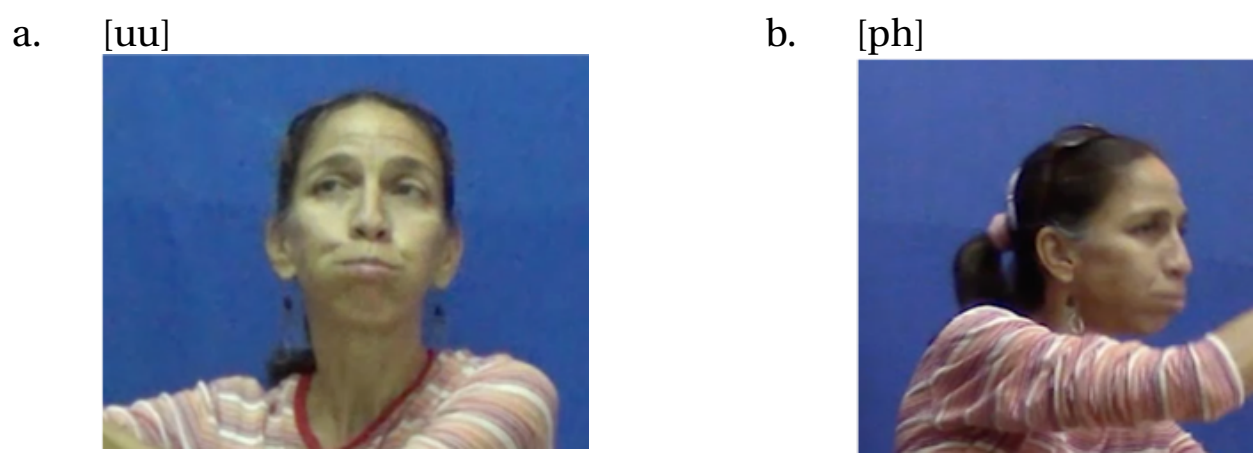

Motion predicates in LSCu, as in many other sign languages, are rendered via Serial Verb Constructions (SVCs), revealing at least 2 predicate sub-events: the process and the telic subevents. In the LSCu example in (12), we can see these two components of an SVC in F.b+IR-VOLAR (for the process) and in F.b+LLEGAR.L (for the telic subevent).

\footnotetext{
${ }^{10}$ This follows standard practices for transcription of language data other than English. We also follow the LSC(u) local tradition of transcribing the sign productions in the local oral language, Spanish.

${ }^{11}$ See also Benedicto and Brentari (2004) and Benedicto, Branchini, and Mantovan (2015) for a systematized listing of the codes used for handshapes.
} 
[0111LSCgIm-a]

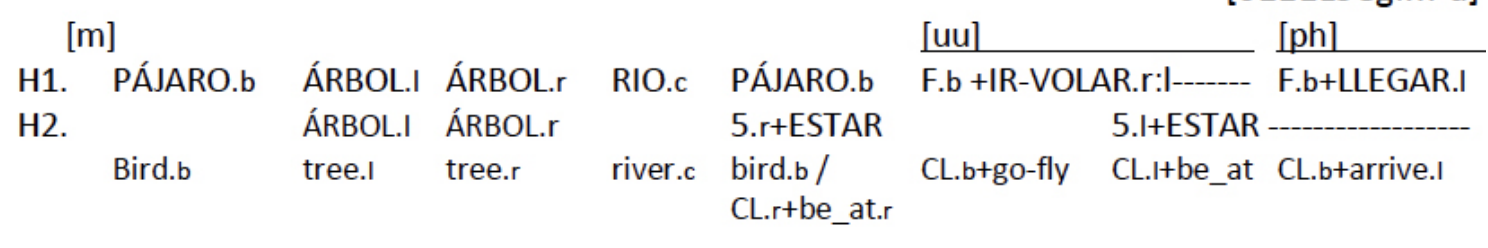

'There is a bird, a tree on the left, a tree on the right, a river in the middle; the bird, at the tree on the right, flies across the river all the way up to the tree on the left.'

The NMMs-[mouth] depicted in (11) correlate with the expression of these two sub-events: [uu] with the process (represented by the manual 3D-Complex Path movement, from right to left); [ph] with the telic REACH (cf. $\pi$ and $\tau$, in (4) and (7)). This can be observed in the transcription in (12), where F.b+IR-VOLAR 'go flying' co-occurs with [uu] and F.b+LLEGAR 'arrive' co-occurs with [ph]. The screenshots in (13) provide detail for this co-occurrence.

a. Process: 3D-Complex Path, $\pi-[\mathrm{uu}]$

H1: F.b+IR-VOLAR

$\mathrm{H} 2:$

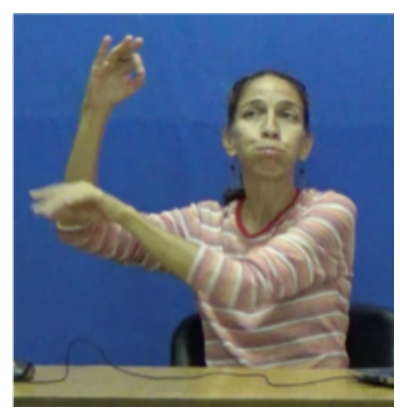

b. Telicity: [REACH], $\tau-[\mathrm{ph}]$

H1: F.b+IR-LLEGAR.l

H2: $5.1+$ ESTAR

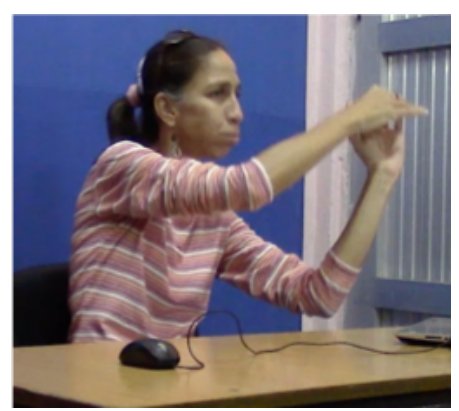

Structure-wise, we will propose that NMM-[mouth] [uu] is part of the morphological characterization of the 3D Complex Head $\pi,{ }^{12}$ which also includes the kinetic movement denoting the displacement of the Figure, the movement from right to left represented by IR-VOLAR 'go-fly'. ${ }^{13}$ Likewise, we will also propose that NMM-[mouth] [ph] is part of the morphological matrix for $\tau$, the telic head REACH, which also includes a short downward movement as part of its manual phonological specification:

\footnotetext{
${ }^{12}$ Alternatively, [uu] could be associated to the functional head $v^{\circ}$, as part of its functional denotation. More research is needed in this respect, at this point.

${ }^{13}$ For details of how that kinetic movement is categorically determined to form the motion vector (the arrow representing the motion from right to left in (12)), see Benedicto, Branchini, and Mantovan (2015).
} 


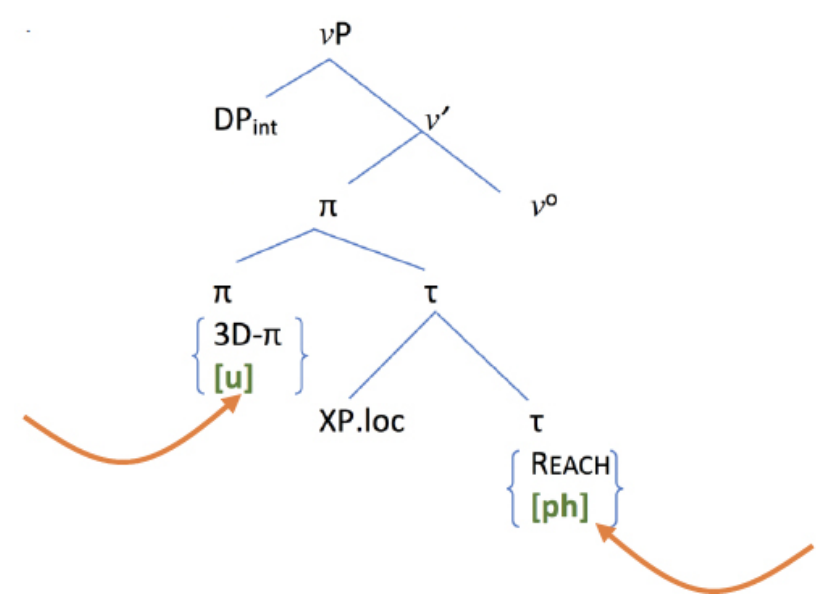

If this structural proposal is in the right track, it predicts that in non-telic utterances [ph] will be necessarily absent since substructure $\tau$, where it belongs, is also absent. On the other hand, since non-telic predicates do have a process subevent, our proposal predicts that [uu] will be present. This is indeed the case, on both counts, as we can observe in example (15):

[0125LSCglm-b]

\begin{tabular}{|c|c|c|c|c|}
\hline$[\mathrm{m}]$ & & & & [uuu] \\
\hline NIÑA.a PÁJARO.b & SOLTAR & F.b+IR-VOLAR & & F.b+IR-VOLARa:/d ------------ \\
\hline bird.b & $\begin{array}{l}\text { SOLTAR } \\
\text { release }\end{array}$ & $\begin{array}{ll}\text { RIO.c } \\
\text { y river }\end{array}$ & $\begin{array}{l}\text { CERCA.d } \\
\text { fence }\end{array}$ & $\begin{array}{l}\text { 4.d+ESTAR ---o--o-d } \\
\text { CL.b+go-fly / CL.d+be_at }\end{array}$ \\
\hline
\end{tabular}

'The girl released the bird across the river towards the fence.'

In (15), the NMM [uu] appears and co-occurs, as expected, with the manual articulation of the movement (F.b+IR-VOLAR, 'go flying'), denoting the process. Since there is no EndPoint ${ }^{14}$ in this utterance, we expect both absence of the manual articulation of REACH (the short downward movement) as well as the NMM [ph], a prediction which is indeed confirm: both are absent in (15).

The structure for [0125LSCglm-b] in (15), thus, is the one in (16) with [uu] under $\pi$, and absence of $\tau$ (with manual REACH and NMM [ph]). In the absence of $\tau$, XP.loc can only be interpreted as 'towards' (and not as 'into').

\footnotetext{
${ }^{14}$ There is a potential Goal, which is CERCA ('fence'), but this does not constitute an EndPoint since it is never reached, that is, the movement does not get to end at the location where CERCA is being articulated.
} 
(16)

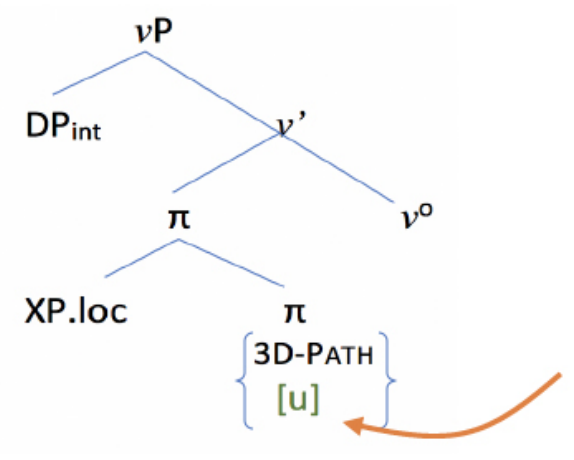

\section{NMM-[eyegaze] patterns}

Let us now address NMM-[eyegaze]. Preliminary analysis of the data indicate that there is [eyegaze] directed at the beginning of the articulation of the classifier predicate when it begins the Path $(\pi)$ movement and that it follows that trajectory; ${ }^{15}$ this is what we see in (17) on F.b+IR-VOLAR 'go-flying'.

There is another [eyegaze] directed at the location of the EndPoint. We see it in (17) on $[ø] . l$, the location in space where the left tree (the purported EndPoint) has been previously articulated, even before the corresponding classifier, 5.1+ESTAR, is articulated at that location (at which point the [eyegaze] returns to the interlocutor). ${ }^{16}$

$$
\begin{aligned}
& \text { [eg] } \\
& \text { [m] } \\
& \text { H1. PÁJARO.b ÁRBOL.I ÁRBOL.r RIO.c PÁJARO.b } \\
& \text { H2. ÁRBOL.I ÁRBOL.r 5.r+ESTAR } \\
& \text { Bird.b tree.l tree.r river.c bird.b/ } \\
& \text { CL.r+be_at.r }
\end{aligned}
$$

[0111LSCgIm-a]

'There is a bird, a tree on the left, a tree on the right, a river in the middle; the bird flies across the river all the way up to the tree on the left.'

The details of those NMM-[eyegaze] can be observed in (18) below:

\footnotetext{
${ }^{15}$ There is, however, no [eyegaze] on the DP Figure as it is initially being set in space on the tree on the right.

${ }^{16} \mathrm{At}$ the point of reaching the EndPoint, the [eyegaze] that had briefly returned to the Path, goes back to the interlocutor. So, while NMM-[mouth] [ph] is being articulated, [eyegaze] is not on the EndPoint but on the interlocutor.
} 
(18)

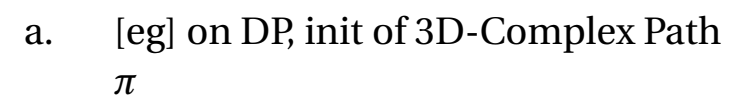

$\pi$

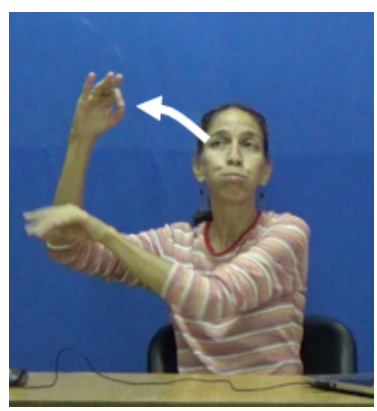

b. [eg] on XP.loc location

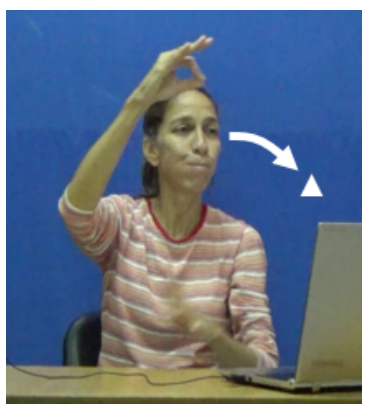

We are going to interpret these instances of NMM-[eyegaze] as instances of agreement, following Neidle et al. (2000). ${ }^{17}$ If so, the structure would look like this:

(19)

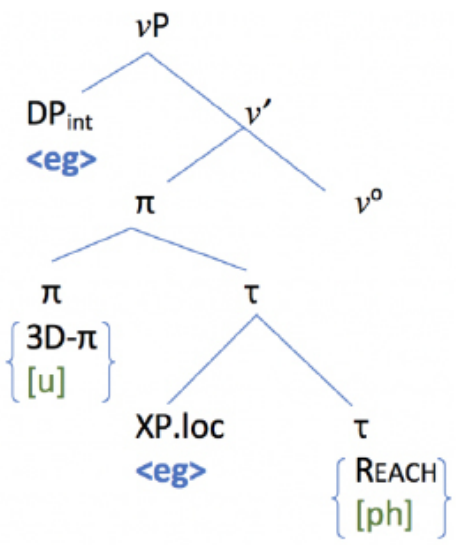

NMM-[eyegaze] would then correlate with the argument of each sub-structure in a motion predicate: the DP (Figure), argument of $v^{\circ}$ (3D-Complex Path and process), and the XP.loc, argument of the telic head $\tau$-REACH. ${ }^{18}$

If our analysis is on the right track, NMM-[eyegaze] on the XPloc is dependent on its interpretation as EndPoint via the presence of the telic $\tau$-REACH head. The prediction, then, is that when that telic $\tau$-REACH head is absent, as in the case of atelic utterances, the XP.loc will not receive the NMM-[eyegaze] (and will not be interpreted as EndPoint, but as mere potential Goal). In the case of (15) (0125LSCglm-b), an atelic utterance repeated here for convenience, this is indeed what we can observe:

\footnotetext{
${ }^{17}$ Thompson, Emmorey, and Kluender (2006), though contesting Neidle et al.'s (2000) claim that eyegaze is agreement on all types of predicates, do find eyegaze on the XP.loc of spatial predicates. However they do not make a distinction between proper EndPoint XP.loc and potential Goal XP.loc, which, under our account, crucially differ with respect to eyegaze, as explained in the discussion about (20).

${ }^{18}$ There is a potential alternative analysis, that could consider NMM-[eyegaze] as Case marking on the arguments rather than Agreement on the predicate. While this is a possibility (especially if we might consider phonologically null arguments, like the EndPoint location we see in (15), [ø].l), at this point, more detailed analysis would be needed to evaluate it.
} 
(20)

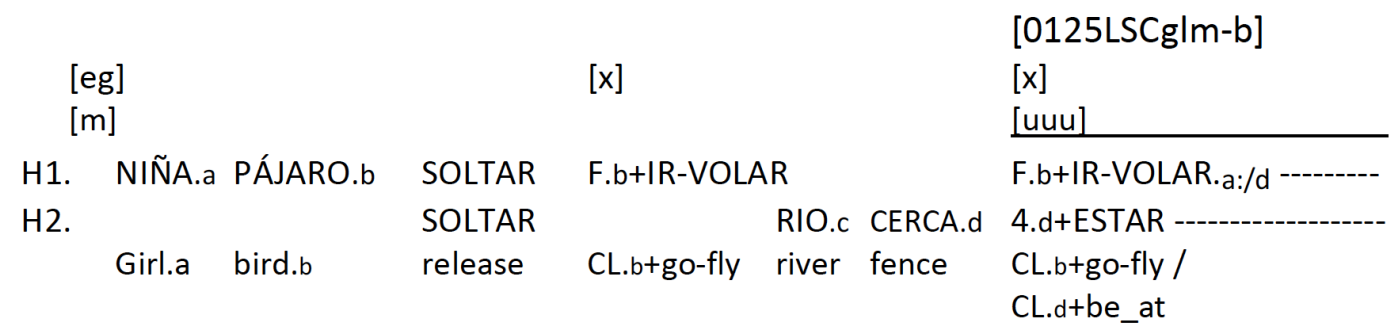

'The girl released the bird across the river towards the fence.'

In (20) we can observe NMM-[eyegaze] on the classifier predicate, F.b+IR-VOLAR 'fly-go', associated with the internal argument DP (the Figure) and its Path (the process). However, the XP.loc CERCA 'fence' does not receive a NMM-[eyegaze], which goes rather to the interlocutor, as we can observe in the screenshots in (21):
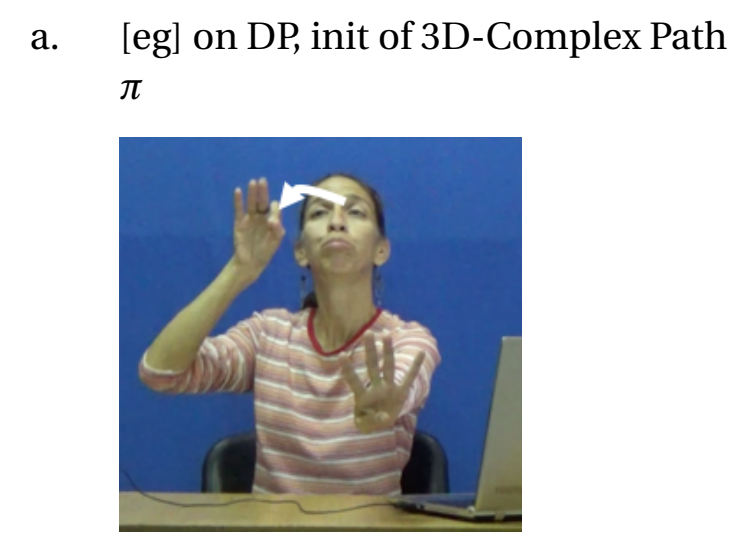

b. [eg] on interlocutor not on XP.loc (CERCA) location (H2)

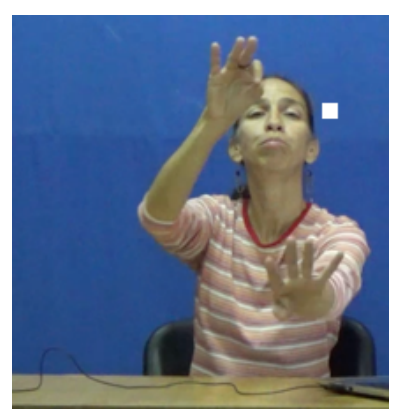

The structure for (20) is thus, that of (16) amended as in (22) to include [eyegaze]: lack of it on XP.loc (correlated with the absence of the telic $\tau$-REACH head) and its presence associated only with the internal argument DP (correlated with the presence of $v^{\circ}+3 \mathrm{D}-\pi$ ):

(22) NMMs for atelic structure

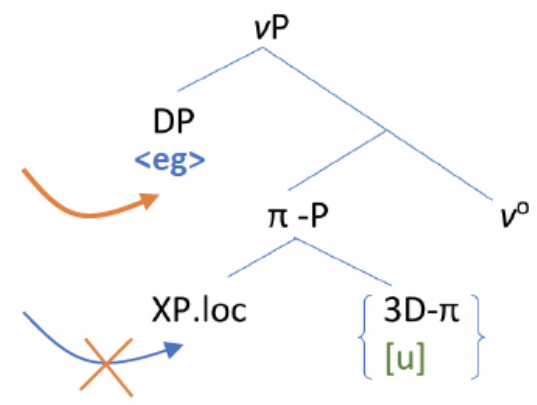




\section{Concluding remarks}

In this paper we have uncovered patterns of systematic association of NMMs for [mouth] and [eyegaze] with the subeventive structure of motion predicates in LSCu. The systematicity of this association has led us to posit their morphological status as part of the complex matrix (including manual and non-manual content) for syntactic heads underlying the structural representation of subeventive components in Motion Predicates.

In particular, thus, we have hypothesized that NMM-[mouth] [uu] is part of the morphological complex forming the 3D-Complex Path head $\pi$, denoting the trajectory and the process subcomponent of the predicate. NMM-[mouth] [ph] is, on the other hand, part of the morphological complex associated to the $\tau$-REACH head, associated to the subcomponent of telicity. With respect to NMM-[eyegaze], we have observed it associated to the internal argument DP (the Figure) as part of the process subeventive component. We have also observed it associated to the XP.loc in the context of the telic subeventive component. We have analyzed these manifestations of NMM-[eyegaze] as instances of agreement as initially postulated in Neidle et al. (2000). The structure that arises from this is the one we summarize in (23):

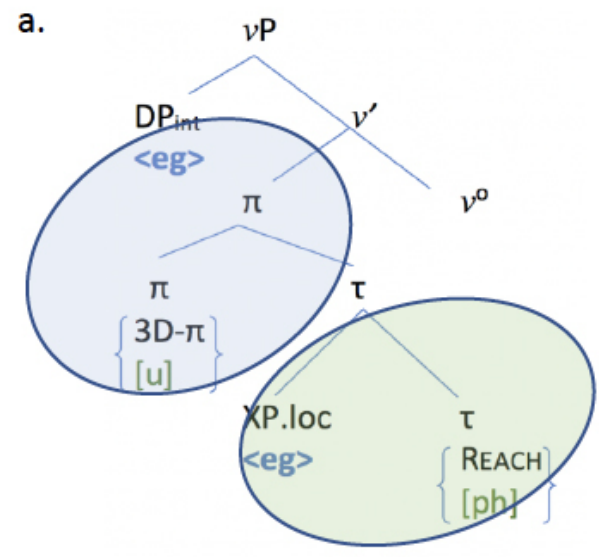

b.

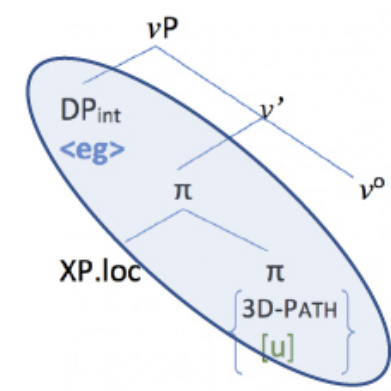

With this paper we contribute to the growing body of literature that provides evidence to show that the Vendlerian Aktionsart categories (Dowty 1979) are actually a product of the syntax of a language and not encoded in the lexical matrix of individual verbs. In particular, the independent availability of a telic head, identified by its NMM [ph] and its associated kinetic properties (quick downward movement), to (freely) combine with process substructures forming SVCs shows that telicity is not an inherent component of lexical entries but part of a syntactic process.

In light of these facts, we can reinterpret the kinetic properties identified in proposals such as the Event Visibility Hypothesis (e.g., Wilbur 2003; Schalber 2006) to reveal complex underlying syntactic structure, rather than lexical verbal classes. Likewise, we can also interpret similar NMM-[mouth] reported in other SLs (e.g. in He and Tang 2018) as indicators of the existence of complex syntactic subeventive structure: they would constitute (part of) the morphemic content of the syntactic subeventive heads proposed in the present paper (on the basis of previous work in Benedicto and Salomon (2014) and Benedicto, Branchini, and Mantovan (2015). 


\section{References}

Aboh, Enoch O. 2009. “Clause structure and verb series”. Linguistic Inquiry 4 (1): 1-33.

Benedicto, Elena. 2017. "Motion predicates: Moving along”. Purdue University Research Repository (PURR), Purdue University. doi:10.4231/R7PN93M4.

Benedicto, Elena, Chiara Branchini, and Lara Mantovan. 2015. "Decomposing the internal structure of motion predicates in Italian Sign Language (LIS)”. Presented at Formal and Experimental Advances in Sign Language Theory (FEAST). Barcelona: Universitat Pompeu Fabra.

Benedicto, Elena, and Diane Brentari. 2004. "Where did all the arguments go?: Argumentchanging properties of classifiers in ASL". Natural Language \& Linguistic Theory 22 (4): 743-810.

Benedicto, Elena, Sandra Cvejanov, and Josep Quer. 2008. “The morphosyntax of verbs of motion in serial constructions: A crosslinguistic Study in three signed languages". In Signs of the time. Selected papers from TISLR 2004, ed. by Josep Quer, 111-132. Hamburg: Signum Verlag.

Benedicto, Elena, and Elizabeth Salomon. 2014. "Multiple V-V mono-eventive syntactic complex in Mayangna”. In Papers from the Seventeenth Workshop on Structure and Constituency in the Languages of the Americas (WSCLA 17), ed. by P. Littell, A. Gutiérrez, R. Girard, and N. Weber, 36:15-27. Vancouver: University of British Columbia Working Papers in Linguistics.

Borer, Hagit. 2005. The normal course of events. Oxford: Oxford University Press.

Calderón, Alicia. 2013. "El valor distintivo del componente manual movimiento en la lengua de señas cubana en su relación con el espacio gestual”. MA thesis, Universidad de La Habana.

Carstens, Vicki. 2002. “Antisymmetry and word order in serial constructions”. Language 78 (1): 3-50.

Chen, Pin-Hsi. 2018. "Motion predicates in Taiwanese Mandarin: Manner, path, and telicity." Unpublished manuscript, Purdue University, West Lafayette, IN.

Collins, Chris. 2002. “Multiple verb movement in キ Hoan”. Linguistic Inquiry 33 (1): 1-29.

Dowty, David. 1979. Word meaning and Montague grammar: The semantics of verbs and times in generative semantics and in Montague's PTQ. Boston: D. Reidel.

He, Jian, and Gladys Tang. 2018. "Causativity and transitivity in classifier predicates in Tianjin Sign Language: a case study”. Presented at Formal and Experimental Advances in Sign Language Theory (FEAST). Venice: Università Ca' Foscari.

Larson, Richard. 1991. "Some issues in verb serialization”. In Serial verbs: Grammatical, comparative and cognitive approaches, ed. by Claire Lefebvre, 185-210. John Benjamins Amsterdam.

Lau, Sin Yee Prudence. 2012. “Serial Verb Constructions in Hong Kong Sign Language”. Doctoral dissertation, The Chinese University of Hong Kong.

Neidle, Carol, Judy Kegl, Dawn MacLaughlin, Benjamin Bahan, and Robert G. Lee. 2000. The syntax of American Sign Language: functional categories and hierarchical structure. Cambridge: MIT Press. 
Osei-Tutu, Kwaku. 2016. "Structuring (A)Telicity in complex-path motion events in Ghanaian Student Pidgin (GSP)”. Unpublished manuscript, Purdue University, West Lafayette, IN.

Ramchand, Gillian. 2008. Verb meaning and the lexicon: A first phase syntax. Cambridge, MA: Cambridge University Press.

Schalber, Katharina. 2006. "Event visibility in Austrian Sign Language”. Sign Language \& Linguistics 9 (1-2): 207-231.

Supalla, Ted. 1990. "Serial Verbs of motion in ASL". In Theoretical Issues in Sign Language Research, ed. by Susan Fischer and Patricia Siple, 1:127-152. Chicago, IL: University of Chicago Press.

Thompson, Robin, Karen Emmorey, and Robert Kluender. 2006. “The relationship between eye gaze and verb agreement in American Sign Language: An eye-tracking study”. Natural Language \& Linguistic Theory 24 (2): 571-604. doi:10. 1007/s11049-005-1829-y.

Vendler, Zeno. 1957. "Verbs and times”. The philosophical review 66 (2): 143-160.

Wilbur, Ronnie. 2003. "Representations of Telicity in ASL”. Proceedings from the Annual Meeting of the Chicago Linguistic Society 39 (1): 354-368. 